\title{
Revisión sistemática exploratoria sobre el intercambio de conocimiento en las comunidades de práctica virtuales
}

\author{
Roberto Hernández-Soto ${ }^{1}$, Mónica Gutiérrez-Ortega ${ }^{1}$ y Bartolomé \\ Rubia-Avi ${ }^{2}$ \\ ${ }^{1}$ Universidad Internacional de la Rioja, España | roberto.hernandez@unir.net; \\ monica.gutierrez@unir.net | https://orcid.org/0000-0002-3505-3108; https://orcid.org/0000- \\ 0002-1536-4240 \\ 2Universidad de Valladolid, España | brubia@pdg.uva.es | https://orcid.org/0000-0002-4963- \\ 4552
}

\begin{abstract}
Resumen: Las Comunidades de Práctica Virtuales (VCOP) son entornos ampliamente reconocidos como instrumentos para la gestión de conocimiento y, de manera incipiente, se están empezando a valorar sus contribuciones desde el punto de vista sociocultural. Sin embargo, las VCOP son contextos de participación complejos por su naturaleza sociotécnica y sociocultural. Los mecanismos de participación, particularmente el comportamiento de compartir conocimiento (KSB), se han abordado desde fundamentos teóricos y métodos de investigación extremadamente heterogéneos. Como resultado, se ha identificado una gran dispersión de factores y dimensiones. Objetivo: Presentar una visión general que resuma y sistematice los factores clave del KSB en las VCOP. Método: Este artículo presenta una revisión sistemática sobre el KSB en las VCOP, basada en 42 estudios recuperados de WOS, SCOPUS y Science Direct. La revisión se ha realizado utilizando el modelo PRISMA. La selección y síntesis cualitativa de los artículos se ha enriquecido utilizando Nvivo para codificar y analizar los artículos a texto completo. Resultados: Los resultados sugieren que el KSB en las VCOP tiene un carácter multidimensional y multifactorial que incluye factores personales, interpersonales, contextuales y tecnológicos. Conclusiones: La tipología de factores que se presenta podría ser utilizada en contextos académicos para desarrollar nuevas investigaciones teóricas o empíricas, o como en contextos profesionales para implementar VCOPs en instituciones de diversos sectores. Nuevos instrumentos de evaluación sobre el KSB en las VCOP podrían basarse en esta tipología.
\end{abstract}

Palabras clave: Comunidades de práctica; Comunidades Virtuales de práctica; Intercambio de Conocimiento; Gestión del Conocimiento; Comportamiento Organizacional; Revisión Sistemática.

\section{Exploratory Systematic Review on the Exchange of Knowledge in Virtual Communities of Practice}

Abstract: Virtual Communities of Practice (VCOP) are environments widely recognized as knowledge management instruments, and their sociocultural contributions are being incipiently valued. However, VCOPs are complex participation contexts due to their sociotechnical and sociocultural nature. Participation mechanisms, particularly Knowledge Sharing Behavior (KSB), have been studied from heterogeneous theoretical foundations and practical research methods. Therefore, a wide dispersion of factors and dimensions has been identified. Goals: Present an overview that summarizes and systematizes the key drivers of KSB in VCOPs. Methods: This paper presents a systematic review of KSB in VCOPs, based on 42 studies retrieved from WOS, SCOPUS and Science Direct. The review was conducted using the PRISMA model. Results: The selection and qualitative synthesis of articles was enriched using Nvivo for coding and analysis of the full text documents. The results suggest that KSB in VCOPs have a multidimensional and multifactorial character that includes personal, interpersonal, contextual, and technological factors. Conclusions: The typology of factors presented could serve in academic settings to conduct new theoretical or empirical research, or in practitioner settings to implement VCOPs in institutions across diverse sectors. New assessment instruments of KSB in VCOPs could be based on this typology.

Keywords: Communities of Practice; VCOP; Knowledge Sharing; Knowledge Management; Organizational Behavior; Systematic Review. 


\section{Introducción}

En el ámbito del desarrollo organizativo, especialmente desde el área de la Gestión del conocimiento (KM; Bolisani \& Scarso, 2014), hay un creciente interés por entender los mecanismos y canales que intervienen en la creación, almacenamiento, recuperación y aprovechamiento del conocimiento (Retna \& Tee, 2011).

En este sentido, las Comunidades de Práctica (COP; Wenger, 1999), se han convertido en un contexto privilegiado para la creación e intercambio de conocimiento (Alali \& Salim, 2013; Wenger et al., 2002; Annabi \& McGann, 2013) y en un mecanismo ampliamente utilizado en KM (Bolisani \& Handzic, 2015).

Las COP son "grupos de personas que comparten una preocupación, un conjunto de problemas o un interés sobre un tema, y que profundizan en su conocimiento y pericia en esta área mediante la interacción continua" (Wenger et al., 2002:4). Aunque en la concepción original las COP eran grupos de carácter informal y autodirigido (Brown \& Duguid, 1991; Wenger, 1999; Lampel \& Bhalla, 2007), su interés para las organizaciones ha ido creciendo, de manera que se han convertido en un activo institucional que debe ser gestionado (McDermott \& Archibald, 2010; Bourhis \& Dubé, 2010) o, al menos, cultivado o promovido por la organización (Jeon et al., 2011a,b). En la actualidad, las COP constituyen un elemento clave del KM, alineando el conocimiento individual con los objetivos institucionales (Retna \& Tee, 2011).

Los avances tecnológicos están permitiendo ampliar el alcance y las capacidades de las COP offline, creando entornos de comunicación y colaboración online efectivos e independientes de las variables espaciotemporales tradicionales (Hildreth et al., 2000). Las Comunidades de Práctica Virtuales (VCOP) han sido descritas por Lee-Kelley y Turner (2017) como comunidades "diseñadas e implementadas como una intervención organizativa, utilizando múltiples plataformas electrónicas síncronas y asíncronas para facilitar el compromiso entre pares a nivel local, de proyecto y organizativo, y el aprendizaje mutuo" (p.66).

En los últimos años, las VCOP son cada vez más relevantes (Bicchi, 2011) no solo porque transcienden la necesidad de proximidad física, sino por su aportación al KM capturando y compartiendo el conocimiento experto de sus miembros, distribuyendo su know-how, ideas, problemas, innovaciones, talentos, y experiencias (Wenger et al., 2002). Más allá del sistema de KM, las VCOP tienen un profundo poder transformador por sus contribuciones desde una óptica sociocultural (Ardichvili et al., 2006; Hou, 2015). En este sentido emergen aspectos como el empoderamiento individual y comunitario (Kirkman et al., 2011), la construcción de la identidad profesional (Nistor \& Fischer 2012; Nistor et al., 2014) o el impacto potencial de la participación en la cultura de la organización o del entorno social (Ardichvili, 2008; Bourhis \& Dubé, 2010), que requieren nuevos enfoques en el estudio de las VCOP.

Tanto en las COP como en las VCOP, el Comportamiento de Compartir Conocimiento (KSB; Tseng \& Kuo, 2014) es uno de los constructos más relevantes y estudiados por su influencia en la viabilidad y en el éxito de este tipo de comunidades (Jeon et al., 2011b). De hecho, Fang y Chiu (2010) señalan que el mayor reto para el funcionamiento de una VCOP es que exista un aporte continuo de conocimiento, por tanto, es de vital importancia conocer los mecanismos implicados en este proceso. El estudio de los factores que impulsan en KSB, va a permitir profundizar en el alcance de las transformaciones que se derivan tanto en el ámbito de la gestión del conocimiento (p.e. aprendizaje y desarrollo individual o mejora organizativa), como en el ámbito sociocultural (p.e. creación de una cultura de la participación y de la colaboración en medios digitales). Sin embargo, existe una enorme dispersión en los enfoques teóricos y metodologías de investigación que se han empleado y, por consiguiente, en los factores y dimensiones que identifican los estudios previos.

Con el fin de aportar una visión integradora, este artículo presenta una revisión sistemática exploratoria (RSE) sobre los factores asociados al KSB en las VCOP. 


\section{Objetivo}

La investigación propuesta tiene como objetivo ofrecer una visión holística del proceso de intercambio de conocimiento en una comunidad de práctica a través de una revisión sistemática exploratoria y síntesis temática de la literatura cuantitativa, cualitativa y mixta centrada en esta temática. Para abordar este objetivo, hay una pregunta de investigación principal: ¿Qué factores intervienen en la conducta de intercambio de conocimiento en el contexto de una VCOP?

La revisión sistemática propuesta se notificará de conformidad con las orientaciones para la presentación de informes proporcionadas en la declaración Mejora de la transparencia en la presentación de informes de la síntesis de investigación cualitativa (ENTREQ) (Tong et al., 2012) y la Declaración PRISMA (Moher et al., 2009).

\section{Metodología}

\subsection{Criterios de Elegibilidad}

Se utilizó la herramienta SPIDER (Sample, Phenomenon of Interest, Design, Evaluation y Research) (Methle et al., 2014) debido a su idioneidad para la síntesis de evidencia cualitativa (Cooke, Smith, \& Booth, 2012). Su utilización apoyó a la definición de la pregunta de investigación y los criterios de elegibilidad.

Los criterios de elegibilidad que se utilizaron en esta investigación fueron todos aquellos artículos que cumplieran con los siguientes criterios de inclusión: (a) se centran en las VCOP (b), analizan los factores que intervienen en el KSB (c) se trata de estudios teóricos o empíricos que utilizan metodologías cualitativas, cuantitativas o mixtas, (d) están publicados en inglés en revistas revisadas por pares.

Se excluyeron resultados sobre otros grupos que no son VCOP (Network of Practice, Research Consortia, Personal Network, Community of Scholars, Occupational Community, Virtual Community).

\subsection{Fuentes de Información y Estrategias de Búsqueda}

En primer lugar, se realizó una búsqueda inicial en las bases de datos Web of Science (WOS), SCOPUS y Science Direct (SD) utilizando los términos "virtual communities of practice" y "knowledge sharing", en inglés y sin límite temporal. Las búsquedas se centraron en el titulo, resumen y palabras clave.

Segundo, con el objetivo de identificar estudios que han investigado las VCOP y el KSB pero que no explicitan estos términos en los campos citados, se realizó una segunda búsqueda complementaria en las citadas bases de datos. Se realizó una búsqueda genérica sobre COP y una posterior depuración manual de los resultados conforme a los criterios de inclusión. Para acotar el número de resultados y asegurar la relevancia de los estudios para la investigación, la búsqueda se restringió a estudios desde el 2010 que tuvieran 10 o más citas en las bases de datos de referencia. La búsqueda se centró en los sectores Social Sciences, Education Educational Research y Business, Management and Accounting.

En la búsqueda sobre VCOP y Knowledge Sharing se han recuperado un total de 176 registros. En la búsqueda sobre COP se han obtenido 1.061 registros, de los cuales 196 cumplían el criterio de tener 10 o más citas.

En la figura 1 se puede observar el flujo seguido en las búsquedas y el proceso de selección. 


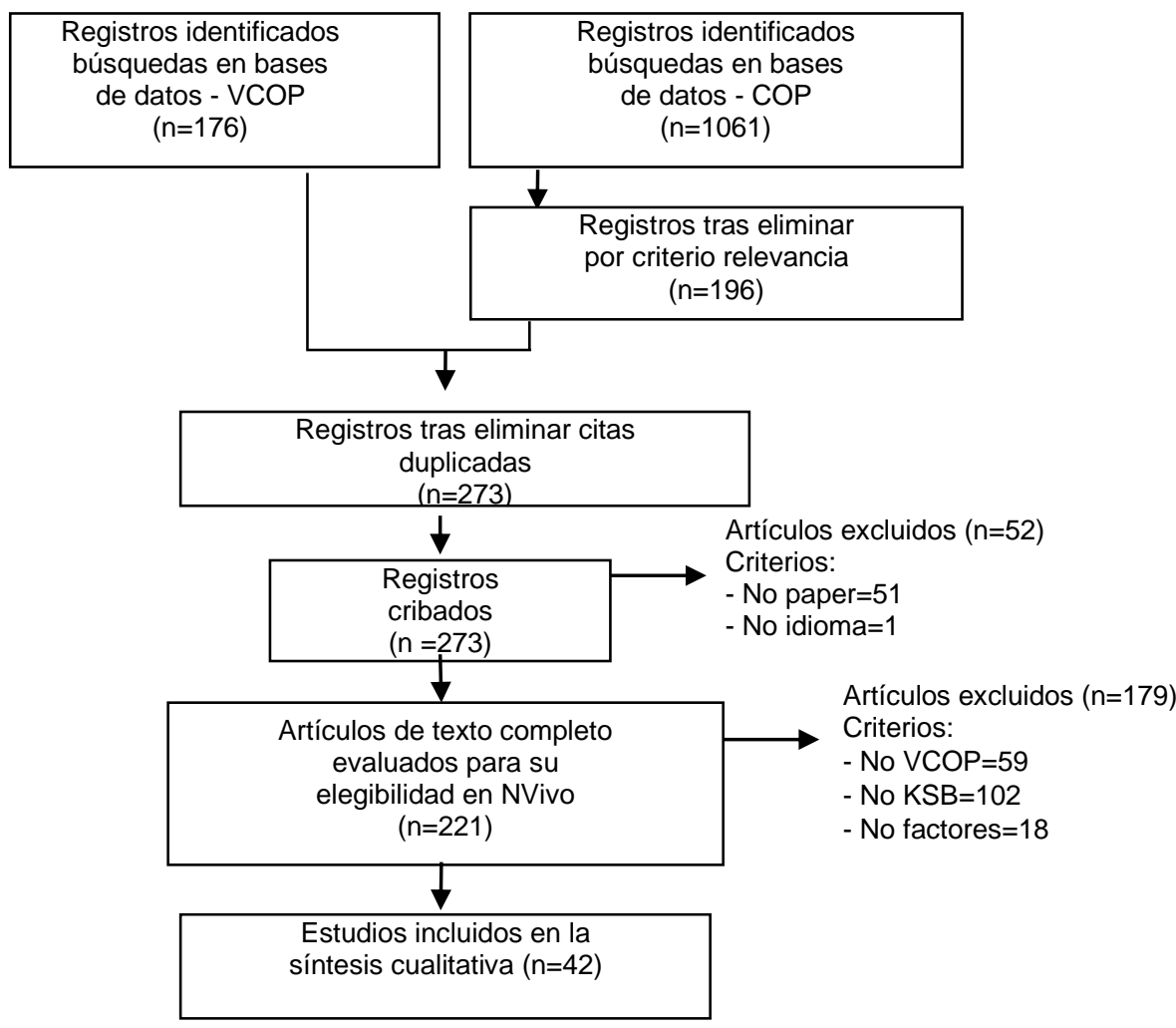

Fig. 1. Diagrama de flujo cuantitativo-cualitativo del proceso de búsqueda y selección del estudio (Moher et al., 2009).

\subsection{Selección de Estudios}

En primer lugar, se eliminaron los registros duplicados. Además, dos investigadores independientes seleccionaron a partir del título y del resumen los artículos que cumplían con los criterios de inclusión. Un total de 273 artículos fueron seleccionados en esta fase.

En caso de desacuerdo, los dos revisores discutieron el estudio para llegar a una decisión sobre la inclusión o exclusión. En caso de que no se llegara a un acuerdo después de la discusión entre los dos revisores, se invitó al tercer revisor a reconciliar su desacuerdo y tomar una decisión final. Después de este proceso de selección, los artículos restantes se incluyeron en la revisión después de la extracción de datos, la evaluación de la calidad y el análisis. Se siguió la declaración PRISMA para crear un diagrama de flujo del número de estudios incluidos y excluidos en cada etapa de este proceso.

Los estudios recogidos se han gestionado en Parsif.al, tras su volcado desde las bases de datos. A continuación, se realizó un análisis de los artículos a texto completo utilizando Nvivo 11.4.3. por dos investigadores independientes y se identificaron finalmente 42 para la síntesis cualitativa. 


\subsection{Extracción de Datos}

Los datos fueron extraídos de los estudios por el primer autor utilizando un formulario de Microsoft Excel probado y diseñado específicamente. Se extrajo información sobre el autor, el año de publicación, la ubicación geográfica del estudio, el enfoque metodológico, el método, la población, y los hallazgos principales para comprender la base de cada estudio. El segundo autor comprobó la exactitud de estos datos extraídos.

Para la síntesis temática, de acuerdo con Thomas y Harden (2008), todo el texto etiquetado como "resultados" o "hallazgos" se extrajo y se introdujo en el software NVivo para su análisis. Esto se realizó porque muchos factores, incluidos los estilos variados de presentación de informes y la tergiversación de los datos como hallazgos, pueden dificultar la identificación de los hallazgos en la investigación cualitativa (Sandelowski \& Barroso, 2002). En consecuencia, se utilizó un enfoque de amplio alcance para capturar tantos datos relevantes como sea posible de cada artículo incluido.

\subsection{Evaluación de la Calidad}

La evaluación de la calidad se ha tenido en cuenta durante el desarrollo de la síntesis de datos. Siguiendo a Soilemezi y Linceviciute (2018), si los autores tuvieron un punto de vista diferente sobre la inclusión o la exclusión de estudios de baja calidad, se introdujeron estudios de menor calidad si ambos autores consideraron que podían agregar hallazgos importantes y de valor para el fenómeno analizado.

\subsection{Síntesis de Datos}

La revisión sistemática actual utilizó la síntesis temática como metodología para crear una comprensión global del proceso de intercambio de conocimiento en las comunidades de práctica virtuales. En la síntesis temática se abordaron temas descriptivos que se mantienen cercanos a los estudios primarios.

A continuación, se emprendió una nueva etapa de desarrollo del tema analítico en la que los revisores van más allá de las interpretaciones de los estudios primarios y desarrollaron constructos de orden superior o explicaciones basadas en estos temas descriptivos (Thomas \& Harden, 2008).

El proceso de síntesis temática para revisión es similar al de la teoría fundamentada para datos primarios, en que se produce una traducción e interpretación de los fenómenos de interés (Glaser \& Strauss, 2017). El uso de la síntesis temática para considerar experiencias subjetivas se fusiona bien con la presente investigación.

Se han seguido las orientaciones de Thomas y Harden (2008) para sintetizar los datos. En primer lugar, el texto extraído se ha codificado de forma inductiva de acuerdo con su contenido y significado. Esta creación inductiva de códigos debe permitir captar el contenido y el significado del texto. Los códigos han sido tanto "libres" como estructurados. Para velar por la calidad de la codificación se ha revisado todo el texto vinculado a un código para garantizar la coherencia o determinar si se requieren más niveles de codificación.

Después de esta etapa, se han examinado las similitudes y diferencias entre los códigos y se comenzó una organización de la jerarquía del grupo de códigos. Cuando fue necesario se aplicaron nuevos códigos a estos grupos para describir su significado general.

Se presenta a continuación la estructura de los temas descriptivos que está alineada con los hallazgos de los estudios incluidos. 
En la etapa final del análisis, se ha reflejado una estructura teórica sobre los factores que influyen en el proceso de intercambio de conocimiento en las comunidades de práctica virtuales (VCOPs). Este proceso inferencial se llevó a cabo mediante la colaboración entre los miembros del equipo de investigación.

Se presenta a continuación los resultados del estudio que muestran que los miembros de una VCOP ejecutan o inhiben el comportamiento de compartir conocimiento en función de aspectos relacionados (1) con sus propias características y creencias o expectativas personales (factores personales), (2) con las relaciones que se establecen entre los miembros (factores interpersonales), (3) con el entorno de referencia para la comunidad (factores contextuales) y (4) con el medio tecnológico en el que se desarrolla la comunicación (factores tecnológicos). La tabla 1 muestra la estructura de categorías, dimensiones y sub-dimensiones o factores que conforman la tipología resultante.

Tabla 1. Tipología de factores asociados al KSB en las VCOP.

\begin{tabular}{|c|c|c|c|}
\hline \multirow{2}{*}{$\begin{array}{l}\text { Categorías } \\
\text { Personales }\end{array}$} & \multicolumn{2}{|l|}{ Dimensiones/Sub-dimensiones } & \multirow{3}{*}{$\begin{array}{l}\text { Factores } \\
\begin{array}{l}\text { Altruismo; Comportamiento } \\
\text { prosocial }\end{array}\end{array}$} \\
\hline & Comportamientos de ayuda & & \\
\hline & $\begin{array}{l}\text { Necesidad de contacto social } \\
\text { pertenencia } \\
\text { Conocimiento experto }\end{array}$ & $\mathrm{y}$ & \\
\hline & Autodirección del aprendizaje & & $\begin{array}{l}\text { Autonomía; Motivación individual } \\
\text { Empoderamiento personal }\end{array}$ \\
\hline & Creencias y validación de expectativas & & \\
\hline & Creencias de ejecución & & $\begin{array}{l}\text { Competencia/eficiencia; relaciones } \\
\text { satisfactorias; esfuerzo; reputación; } \\
\text { recompensas; reciprocidad; calidad } \\
\text { del conocimiento }\end{array}$ \\
\hline & Creencias normativas & & Influencia social \\
\hline & Creencias de control & & $\begin{array}{l}\text { Auto-eficacia; restricciones } \\
\text { externas }\end{array}$ \\
\hline & Validación de expectativas & & $\begin{array}{l}\text { Reciprocidad; ayuda a otros; } \\
\text { utilidad del conocimiento; calidad } \\
\text { información }\end{array}$ \\
\hline \multicolumn{4}{|c|}{ Interpersonales } \\
\hline & Confianza y justicia & & Confianza; Justicia \\
\hline & Liderazgo & & \\
\hline & Vínculos personales & & \\
\hline & Estatus de experto & & \\
\hline & Empoderamiento de la VCOP & & \\
\hline & Estructura relacional de la VCOP & & $\begin{array}{l}\text { Conectividad; Interactividad; } \\
\text { Participación inclusiva; Comunidad } \\
\text { diversa }\end{array}$ \\
\hline \multicolumn{4}{|c|}{ Contextuales } \\
\hline & Comunidad (como contexto) & & Dominio; Apertura; Autonomía \\
\hline & $\begin{array}{l}\text { Organización de referencia } \\
\text { Contexto cultural }\end{array}$ & & Cultura; Soporte \\
\hline \multicolumn{4}{|c|}{ Tecnológicos } \\
\hline & Individuales & & Experiencia de uso \\
\hline & Técnicos & & Calidad del sistema \\
\hline & Contextuales & & Soporte tecnológico \\
\hline
\end{tabular}

\section{Conclusiones}

La revisión sistemática propuesta contribuirá al conocimiento sobre que factores intervienen en el proceso de intercambio de conocimiento en las VCOPs. Sintetizar la literatura cualitativa, cuantitativa y mixta que se centra en el objeto de estudio ofrece una comprensión derivada de los componentes de este fenómeno. 
Al incluir poblaciones de diferentes sectores (empresarial, educativo, salud, tecnología), esta investigación proporciona una amplia perspectiva sobre los mecanismos de intercambio de conocimiento en las VCOP y sugiere que dicho comportamiento depende de la interrelación dinámica de una amplia gama de factores personales, interpersonales, contextuales y tecnológicos.

Creemos que la nueva tipología que se presenta permite identificar los factores que intervienen en el KSB en las VCOP y conocer cómo se organizan y cómo se relacionan. Partiendo de la misma, se presentan múltiples líneas de investigación orientadas a la evaluación de la situación de una VCOP o a la creación de ecosistemas inclusivos que favorezcan la aparición de comunidades emergentes o que den soporte a las comunidades formales.

Los resultados de la RS indican que el constructo KSB tiene un carácter multidimensional y multifactorial. La tipología de factores presentada ofrece una amplia perspectiva sobre los mecanismos de intercambio de conocimiento en las VCOP. Los hallazgos permiten extraer las siguientes conclusiones.

Primero, el intercambio de conocimiento (KS) en las VCOP depende ampliamente de factores personales como las motivaciones internas, las creencias y la evaluación de las expectativas de los miembros sobre su experiencia en la comunidad. Efectivamente, los comportamientos altruistas y procomunitarios emergen como precursores del KS que prevalecen sobre motivaciones externas como la obtención de incentivos materiales o la mejora de la reputación (Jeon et al., 2011b). En cuanto a las creencias, los miembros comparten su conocimiento cuando prevén consecuencias positivas (p.e. desarrollo competencial o mejora de su red profesional), cuando perciben una presión social hacia el KS, y cuando creen que su conocimiento puede ayudar a otros (percepción de autoeficacia). Finalmente, la intención de seguir compartiendo conocimiento depende de la satisfacción de los miembros con la calidad de la información y utilidad práctica del conocimiento que se comparte, con la reciprocidad en los intercambios, y con su capacidad de ayudar a otros con su conocimiento.

Segundo, en el contexto sociotécnico y sociocultural de las VCOP, los elementos personales convergen en una compleja red de relaciones interpersonales que resulta determinante en el KSB. Además, los estudios previos revelan una relación de mutua influencia entre los factores personales e interpersonales. En esta línea, Fang y Chiu (2010) sitúan la confianza en los miembros como antecedente del altruismo, mientras que la confianza en los líderes de la comunidad sería el determinante principal del comportamiento procomunitario (Ardichvili, 2008; Hernández-Soto, Rodríguez-Medina, \& Gutiérrez-Ortega, 2020) Igualmente, la existencia de vínculos personales incrementa los comportamientos altruistas y la percepción de autoeficacia sobre el propio conocimiento para ayudar a otros, por lo que las relaciones personales intervienen de manera decisiva en la construcción y desarrollo de la comunidad (Usoro \& Majewski, 2011). En cuanto sus líderes, además de generar confianza, ejercen una labor clave alineando la participación con los objetivos institucionales (especialmente en las comunidades formales), regulando y facilitando la participación, y fomentando el compromiso de los miembros (Barnett et al., 2014).

Tercero, los resultados avalan el rol prominente de los factores contextuales como determinante clave del KSB en las VCOP. En este sentido, las políticas institucionales (p.e. normativa, procedimientos y valores), las políticas de recursos humanos (p.e. sistemas de reconocimiento y promoción) y el estilo de liderazgo corporativo, son elementos claves en la creación de una cultura de intercambio de conocimiento. Esta cultura actúa como facilitador KSB en la medida que promueve y valora la participación, y cuenta con el apoyo de los líderes de la organización. En la dirección opuesta, la participación en la VCOP tiene un profundo potencial transformador en la cultura individual que, a su vez, se traslada a su entorno institucional y social de referencia (Ardichvili et al., 2006; Hou, 2015). 
Cuarto, como parte de la cultura institucional, el empoderamiento de los miembros y de la propia comunidad, confiere a las VCOP una especial relevancia en la creación de una cultura de la participación y del compromiso de sus miembros con la mejora individual y organizativa (Retna \& Tee, 2011). En el plano individual, la VCOP demanda y genera dinámicas de participación que promueven una actitud proactiva ante el aprendizaje en contextos fuertemente mediados por la tecnología; en el plano comunitario, la conciencia colectiva sobre la relevancia organizativa de la VCOP (empoderamiento de la comunidad) alienta la participación e incrementa significativamente su influencia en los resultados institucionales. Por tanto, el KS en las VCOP, requiere que los miembros adopten un papel proactivo, comprometido y responsable con el proceso de aprendizaje individual y colectivo, lo que les permite convertirse en agentes de cambio e impulsar su competitividad y la de su organización. El potencial transformador de las VCOP reside, por tanto, en transcender su naturaleza como sistema de aprendizaje social, para convertirse en una palanca de cambio social y organizativo.

Quinto, el uso de medios sociales (como Twitter), digitales (como foros, wikis, intranets, o plataformas de videoconferencia), u otras tecnologías emergentes como los Immersive Virtual Worlds (IVW), está permitiendo crear VCOPs que transcienden los límites espacio-temporales de las comunidades tradicionales y genera nuevas formas de participación, de aprendizaje y de colaboración.

Finalmente, si bien hay un interés creciente, aún existe un número limitado de estudios sobre la influencia de los aspectos culturales en el KSB por lo que se considera una línea futura de investigación de gran relevancia en este campo. Además, investigaciones futuras deberían abordar el potencial transformador de la participación en las VCOPs en los elementos culturales de distintos contextos organizativos y sociales.

\section{Referencias}

Alali, H., \& Salim, J. (2013). Virtual communities of practice success in healthcare sector. Applied Mechanics and Materials, 411, 950-953. https://doi.org/10.4028/www.scientific.net/AMM.411414.950

Annabi, H., \& McGann, S. T. (2013). Social media as the missing link: Connecting communities of practice to business strategy. Journal of Organizational Computing and Electronic Commerce, 23(1-2), 56-83. https://doi.org/10.1080/10919392.2013.748608

Ardichvili, A. (2008). Learning and Knowledge Sharing in Virtual Communities of Practice: Motivators, Barriers, and Enablers. Advances in Developing Human Resources, 10(4), 541554. https://doi.org/10.1177/1523422308319536

Ardichvili, A., Maurer, M., Li, W., Wentling, T., \& Stuedemann, R. (2006). Cultural influences on knowledge sharing through online communities of practice. Journal of Knowledge Management, 10(1), 94-107. https://doi.org/10.1108/13673270610650139

Barnett, S., Jones, S. C., Caton, T., Iverson, D., Bennett, S., \& Robinson, L. (2014). Implementing a virtual community of practice for family physician training: A mixed-methods case study. Journal of Medical Internet Research, 16(3), e83. https://doi.org/10.2196/jmir.3083

Bicchi, F. (2011). The EU as a community of practice: foreign policy communications in the COREU network. Journal of European Public Policy, 18(8), 1115-1132. https://doi.org/10.1080/13501763.2011.615200

Bolisani E., \& Scarso, E. (2014). The place of communities of practice in knowledge management studies: a critical review. Journal of Knowledge Management, 18(2), 366-381. https://doi.org/10.1108/JKM-07-2013-0277

Bolisani, E., \& Handzic, E. (Eds) (2015). Advances in Knowledge Management: Celebrating Twenty Years of Research and Practice. Springer.

Bourhis, A., \& Dubé, L. (2010). «Structuring spontaneity»: Investigating the impact of management practices on the success of virtual communities of practice. Journal of Information Science, 36(2), 175-193. https://doi.org/10.1177/0165551509357861 
Brown, J.S., \& Duguid, P. (1991). Organizational learning and communities of practice: Towards a unified view of working learning and innovation. Organization Science, 2(1), 40-57. https://www.jstor.org/stable/2634938

Cooke, A., Smith, D., \& Booth, A. (2012). Beyond PICO: the SPIDER tool for qualitative evidence synthesis. Qualitative health research, 22(10), 1435-1443. https://doi.org/10.1177/1049732312452938

Fang, Y. H., \& Chiu, C. M. (2010). In justice we trust: Exploring knowledge-sharing continuance intentions in virtual communities of practice. Computers in Human Behavior, 26(2), 235-246. https://doi.org/10.1016/j.chb.2009.09.005

Glaser, B. G., \& Strauss, A. L. (2017). Discovery of grounded theory: Strategies for qualitative research. Routledge.

Hernández-Soto, R., Rodríguez-Medina, J. \& Gutiérrez-Ortega, M. (2020). Trust and knowledge sharing in a transdisciplinary community of practice: a convergent parallel case study. Revista Latinoamericana de Tecnología Educativa, 19(2), 47-63. https://doi.org/10.17398/1695288X.19.2.47

Hildreth, P., Kimble, C., \& Wright, P. (2000). Communities of practice in the distributed international environment. Journal of Knowledge Management, 4(1), 27-38, https://doi.org/10.1108/13673270010315920

Hou, H. (2015). What makes an online community of practice work? A situated study of Chinese student teachers' perceptions of online professional learning. Teaching and Teacher Education, 46, 6-16. https://doi.org/10.1016/j.tate.2014.10.005

Jeon, S.-H., Kim, Y.-G., \& Koh, J. (2011a). Individual, social, and organizational contexts for active knowledge sharing in communities of practice. Expert Systems with Applications, 38(10), 12423-12431. https://doi.org/10.1016/j.eswa.2011.04.023

Jeon, S., Kim, Y. G., \& Koh, J. (2011b). An integrative model for knowledge sharing in communities-of-practice. Journal of Knowledge Management, 15(2), 251-269. https://doi.org/10.1108/13673271111119682

Kirkman, B. L., Mathieu, J. E., Cordery, J. L., Rosen, B., \& Kukenberger, M. (2011). Managing a New Collaborative Entity in Business Organizations: Understanding Organizational Communities of Practice Effectiveness. Journal of Applied Psychology, 96(6), 1234-1245. https://doi.org/10.1037/a0024198

Lampel, J., \& Bhalla, A. (2007). Let's get natural: The discourse of community and the problem of transferring practices in knowledge management. Management Decision, 45(7), 1069-1082. https://doi.org/10.1108/00251740710773916

Lee-Kelley, L., \& Turner, N. (2017). PMO managers' self-determined participation in a purposeful virtual community-of-practice. International Journal of Project Management, 35(1), 64-77. https://doi.org/10.1016/j.ijproman.2016.09.014

McDermott, R., \& Archibald, D. (2010). Harnessing your staff's informal networks. Harvard Business Review, 88(3), 82-89.

Methley, A. M., Campbell, S., Chew-Graham, C., McNally, R., \& Cheraghi-Sohi, S. (2014). PICO, PICOS and SPIDER: a comparison study of specificity and sensitivity in three search tools for qualitative systematic reviews. BMC health services research, 14(1), 1-10. 10.1186/s12913014-0579-0

Moher, D., Liberati, A., Tetzlaff, J., Altman, D. G., \& The PRISMA Group. (2009). Preferred Reporting Items for Systematic Reviews and Meta-Analyses: The PRISMA Statement. PLoS Medicine, 6(7), e1000097. http://doi.org/10.1371/journal.pmed.1000097

Nistor, N., \& Fischer, F. (2012). Communities of practice in academia: Testing a quantitative model. Learning Culture and Social Interaction, 1(2), 114-126. https://doi.org/10.1016/j.Icsi.2012.05.005

Nistor, N., Baltes, B., Dascălu, M., Mihăilă, D., Smeaton, G., \& Trăuşan-Matu, Ş. (2014). Participation in virtual academic communities of practice under the influence of technology acceptance and community factors. A learning analytics application. Computers in Human Behavior, 34, 339-344. https://doi.org/10.1016/j.chb.2013.10.051 
Retna, K. S., \& Tee, P. N. (2011). Communities of practice: Dynamics and success factors. Leadership \& Organization Development Journal, 32(1), 41-59. https://doi.org/10.1108/01437731111099274

Sandelowski, M., \& Barroso, J. (2002). Finding the findings in qualitative studies. Journal of nursing scholarship, 34(3), 213-219.

Soilemezi, D., \& Linceviciute, S. (2018). Synthesizing qualitative research: reflections and lessons learnt by two new reviewers. International Journal of Qualitative Methods, 17(1), https://doi.org/10.1177/1609406918768014

Thomas, J., \& Harden, A. (2008). Methods for the thematic synthesis of qualitative research in systematic reviews. BMC medical research methodology, 8(1), 1-10. doi:10.1186/1471-22888-45

Tong A, Flemming K, Mclnnes E, Oliver S., \& Craig J. (2012). Enhancing transparency in reporting the synthesis of qualitative research: ENTREQ. BMC Med Res Methodol,12(1). https://doi.org/10.1186/1471-2288-12-181

Tseng, F.-C., \& Kuo, F.Y. (2014). A study of social participation and knowledge sharing in the teachers' online professional community of practice. Computers \& Education, 72, 37-47. https://doi.org/10.1016/j.compedu.2013.10.005

Usoro, A., \& Majewski, G. (2011). Intensive knowledge sharing: Finnish Laurea lab case study. VINE, 41(1), 7-25. https://doi.org/10.1108/03055721111115520

Wenger, E. (1999). Communities of practice. Learning, meaning, and identity. University Press.

Wenger, E., McDermott, R., \& Snyder, W. (2002). Cultivating communities of practice: A guide to managing knowledge. Harvard University Press. 